\title{
The role of street traffic signs in reducing road accidents
}

\author{
H. A. Hussein \\ Department of Architectural Engineering, Koya University, \\ Kurdistan Region, Iraq
}

\begin{abstract}
The city roads network is considered an essential element in any urban community. It occupies around $30 \%$ of the total area of urban land. These roads might be classified into two main parts:

- Those which provide services to the lands attached.

- Those which provide services to the cars; they are used by large-size vehicles.

Though the first one is the most common one, the second one has recently been more influential in the planning of a city's roads network as a result of the increased use of high-speed comfortable low cost transportation. Statistics in many countries, including Iraq, have demonstrated that road accidents continue to be an outstanding danger to humankind, which is a problem that requires a study to the reasons behind it. The poor planning of the traffic networks including the traffic lights, the drivers' legal binding, the general level of education that both drivers and pedestrians have, and the correct choice of appropriate transportation suitable for all types of roads are only some examples. Due to the comprehensive nature of the study, it is useful to limit it to a very restricted part of the problem, though with great influence in the traffic process and planning, so this study is about traffic lights with lines and signs that have not received any attention in our country despite their low-cost execution. Keywords: transportation, traffic accidents, traffic signals, streets.
\end{abstract}

\section{Introduction}

Over the long suffering of man in his quest to move between different places and meet the conditions of harsh weather and he terrain rugged; started to think to overcome these difficulties of slowly moving and difficulty to transport the 
different needs; through that atmosphere and terrain to innovate and transport; began urgency to use carts pulled by different animals, and ended with modern modes of transport. First and foremost, land vehicles to distinguish them easily to use, appropriate cost compared to other types. As far as I brought these media huge benefits were found and significant negative results, including environmental pollution, traffic accidents, which needs to review some aspects. Street traffic signs are largely neglected by the official authorities, as well as the drivers of vehicles.

\subsection{Transport and communications}

It is the process of transporting people and goods from one place to another, transportation take people to places they want to go to, and come to them with goods they need or desire. There are three main types: Overland, water, and air. Road transport depends on vehicles with wheels, especially the cars, trucks and trains. The ships and boats are the most important water transport. It is also depends on air transport using aircraft entirely.

\subsection{Traffic accidents}

Traffic accident is interceptor event happening without prior planning by one car (vehicle) or more. This usually results in traffic accidents which vary from minor damage to property vehicles to serious disability or death.

\section{Rules and traffic signals}

The traffic became with serious complex issues, therefore, it is important that every driver must be aware of traffic rules and must comply fully with them. Here is a clarification of the signaling systems used in organizing traffic:

a- Optical signals

b- Traffic police signals

c- Signals performed by the driver

d- The lines on the surface of the road

As mentioned, the low-cost terrestrial signals play an important role in reducing traffic accidents. We must here install what is internationally recognized; in order to highlight the possibility of drawing attention to them and putting them in necessary places for the safety of traffic. Marking the ground is one of the tools to organize the traffic, using paints or buttons or other tools which are placed on the surface of the road in order to give drivers information and orientation, or warn them or guide them as they drive on the roads. They may be used alone or as complementary tools for organizing traffic. 


\subsection{Paints}

The more material signs commonly used are paints applied to the installed materials to help stability and prolong life. These paints may be normal or thermal or rubber strips which are affixed to the surface of the road and preferably reflective light at night by adding small glass crystals sprayed.

\subsection{Buttons}

These buttons are used with a landline or a substitute for, and are arranged in a manner proportionate to the fonts used, whether solid lines or dashed lines.

Features of these buttons:

- Be in the form of circular, square or rectangle shape, and be at height of less than $25 \mathrm{~mm}$.

- You can use the buttons light reflective jointly with non-reflective signs of light.

- These buttons are reflective of light in order to provide sufficient vision for the driver on the road. Yellow and appropriate buttons are used to denote the edges of the road, while the white clickable interface used to denote lanes.

- Take these buttons font colors that are on them.

- Colors can be either yellow or white.

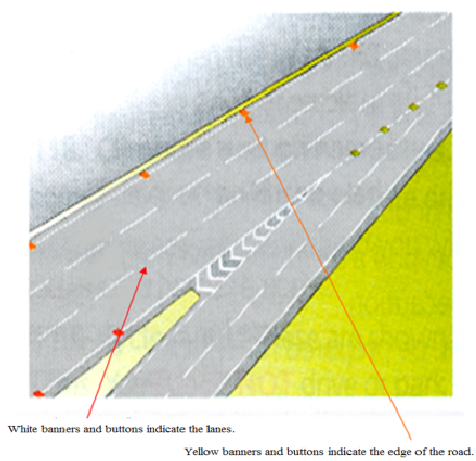

Figure 1: $\quad$ Street banners and buttons.

\subsection{Lines}

They may be at longitudinal, transverse or oblique angle, as they may be connected lines to prevent or intermittent warning or guidance, and each of these lines will refund certain connotations as mentioned later (see Figure 2).

\subsection{Symbols}

The most important signal which indicates the compulsory direction of the lanes placed, either forward or to the right or to the left or to the front with the right or the left. 


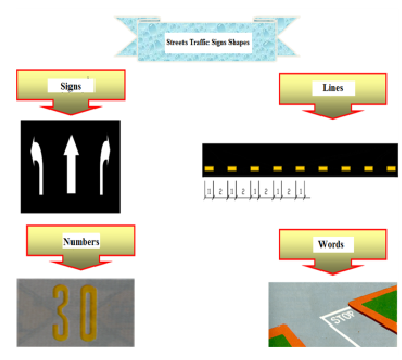

Figure 2: $\quad$ Street traffic signs.

\subsection{Words}

Words like STOP are sometimes used to be complementary to the existing message inside fastened Stand and help to understand the symbols more.

\subsection{Numbers}

The numbers are written on the road surface and typically show the maximum speed limits on the road.

\subsection{Marks' locations}

Either on the road surface such as longitudinal lines related to intermittent or occasional, such as fonts, italics, and often aim to separate traffic direction or prevent overtaking, or it may be on the sidewalks or islands, which are usually placed to guide drivers.

\subsection{Types of land marks}

- Obligatory marks;

- Continuous longitudinal lines;

used for the following:

- Isolate the flow of traffic;

- Identify areas of non-overtaking;

- When the road will be narrow or expand;

- Upon arrival to the impediments in the middle of the road.

\subsection{Occasional lines}

Stop line: The continuous line is white, where you must stop until traffic moves. It is used alone or is complementary to marks. We can write words like STOP before this line. The line width is $30 \mathrm{~cm}$ and can be $50 \mathrm{~cm}$ on wide roads with high speeds. 


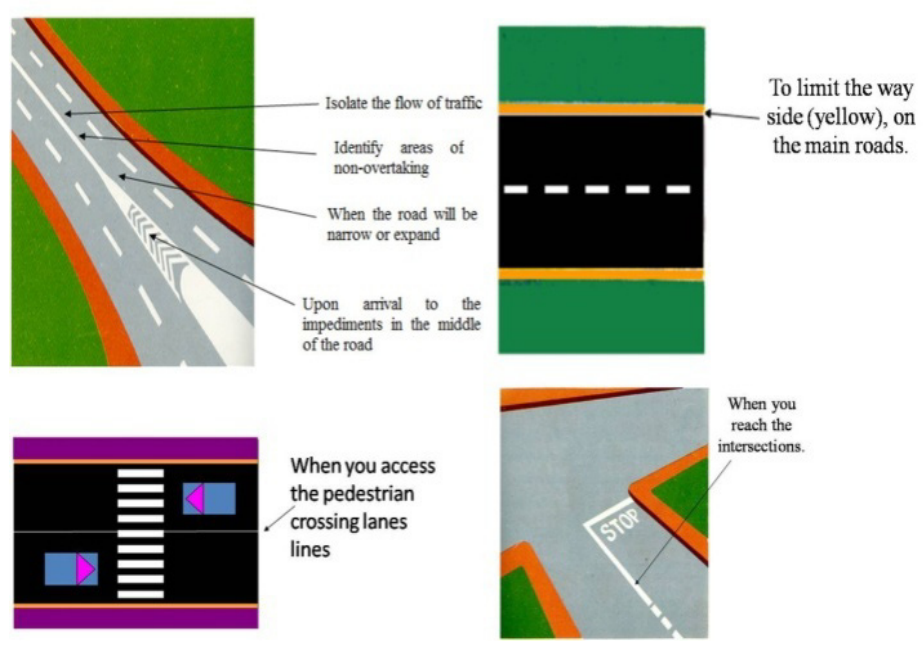

Figure 3: $\quad$ Types of marks.

\subsection{Lines of priority}

The dashed line in the form of small triangles are used to indicate the point at which the driver stands then when necessary to give priority, used alone or be complementary to give priority (inverted triangle).
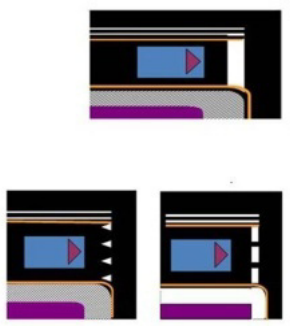

Figure 4: $\quad$ Types of priority marks.

\subsection{Lines obstacles}

A traffic paint islands in front of the barriers be slanted or full paint and surrounded by mandatory lines or warning.

\subsection{Pedestrian crossing lanes lines}

Lines which refer to the place designated for pedestrian crossing the road, and are commonly used lines before reaching the winding footpaths to alert the driver of approaching the crosswalk. 


\subsection{Bike paths lines}

Two lines and there is figure for a bicycle between them, or be dotted lines to define the limits of bicycle path, and give priority for bicycles (Figure 5).

\subsection{Words and numbers}

Words are used to identify the uses of lanes and situations are or to indicate the rule that must be adhered to by the driver at dangerous intersections, which applies when there is a STOP sign. The numbers placed to indicate the maximum speed limits set on the road (Figure 5).
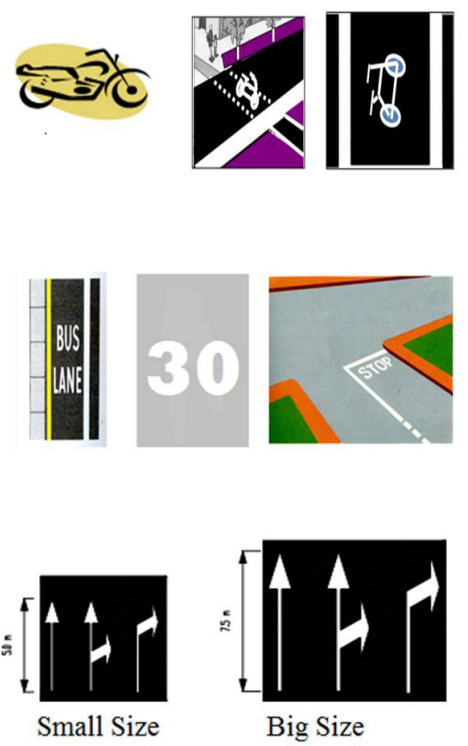

Figure 5: $\quad$ Types of marks.

\subsection{Direction arrows}

- Indicate the direction compulsory as indicated by the arrows, and the effect is similar to the effect of compulsory marks trend mandatory blue.

- Be located in the middle of the user lane.

- If speed is more than 60 miles per hour these stocks are large size.

- Always take the white color.

- Often the mandatory ground signs are placed together such as the continuous stop line next to the word (STOP) in order to increase clarity and stress on being compulsory to the driver. 


\subsection{Longitudinal double lines}

a. The presence of one or two solid lines which refer to the same meaning, never exceed them.

b. When there are two lines, one is solid and the other is intermittent, then it is forbidden to bypass the solid line if it is on the side of the vehicle, but if the line adjacent to the vehicle is an intermittent, then overtaking is allowed. These lines are different in the ratio of the length of the space to the line depending on whether they are inside or outside of the cities, as follows:

A vehicle may overtake since it is adjacent to the dotted line, whereas the vehicles located in the opposite direction cannot overtake being adjacent to the solid line.

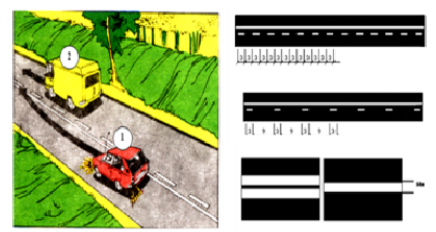

Figure 6: Longitudinal double lines.

\subsection{Meandering line}

This line is used by the crosswalk to confirm the vision of the pedestrian crossing lanes and to indicate that there are no places to stand before these lines. The color of this yellow line and fixed-length according to the specifications and is used when the distance before the crosswalk is not less than 4 meters and not more than 20 meters (Figure 7(a)).

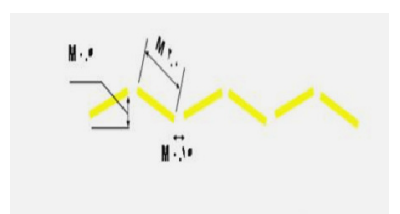

(a)

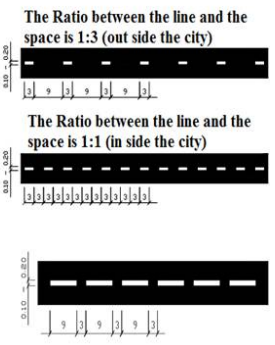

(c)

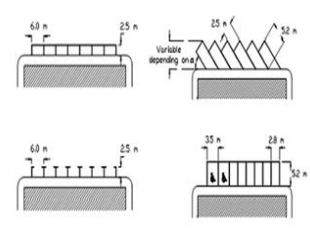

(b)

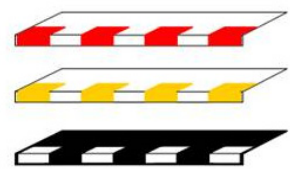

(d)

Figure 7: $\quad$ Types of marks. 


\subsection{Parking lines}

They are to limit the parking lots must stand always within the white lines, and these lines are set out below (see Figure 7).

\subsection{Warning signs}

They are intermittent longitudinal lines so that the ratio of the length to the length of space is $1: 3$ which is used when the driver intends to warn the driver that he take care and reduces the speed.

\subsection{Sidewalks signs}

Sidewalks can be painted excerpts from white or red or yellow illustrating the meaning of the different signs in colors of sidewalks.

Red color: foreclosed stand for all vehicles.

Yellow: The position is only for public transport vehicles (bus and taxi).

White: to confirm the vision to make the sidewalks more pronounced.

\section{Final conclusions}

- The traffic problem has complex aspects, involving elements of various destinations, starting from the planners of cities; and implementing roads and culture of drivers and citizens ... all of which complete each other.

- But the traffic problem which remains; is the time shortage for the driver to react due to the high speed. To overcome this problem we may either increase this time by giving the driver an early warning signal or exploit the available time through taking the necessary reaction in a simple and clear way. In both cases the solution is in adopting the ground traffics signs and lines.

- Recognition on the international traffic signs trying to apply them in the country for the purpose of utilization the experiences of developed countries.

- Work to raise the cultural level of the users of public streets, drivers, cyclists and pedestrians.

- Paying most attention to the quality of ground traffic signs.

- Putting severe legal consequences and anyone who violates traffic laws and reconsidering the law of traffic fines.

\section{References}

[1] Petter Naess, Urban structure matters: residential location, car dependence and travel behaviour, Routledge, Oxon, 2006.

[2] Michael Southworth, Streets and the shaping of town and cities, Eran BenJoseph, Island Press, Washington, 2009.

[3] Lewis Keeble: Principal and Practical of Town and Country, London, 1981.

[4] William L. Pereira, New District of Doha Development Plan, Doha 1982. 
First International Symposium on Urban Development: Koya as a Case Study 311

[5] Hamid Abdul Wahid/Introduction to Urban Transportation Planning, Doha, 1985.

[6] Abdel Fattah Mohamed Wahiba, Geographical Inhabitedness, Beirut, 1980. 